\title{
Retrospective Comparison of Traditional and Enhanced Recovery After Surgery Protocols Among Women Undergoing Mastectomy
}

Walid Abou-Jaoude, MD, Thomas J. Young, MB, John M. Edwards III, MS, CRNA, Stace D. Dollar, MS, CRNA, Heather Shearin, MS, CRNA, Barbara Self, BS, RN, OCN, Stacy Stanifer, APRN, MSN, AOCNS, Susan Yackzan, PhD, APRN, AOCN

\section{Background}

Patients undergoing mastectomy may encounter problems with pain management and postoperative nausea and vomiting, hindering their recovery. We developed an ERAS protocol in an attempt to address these problems. The purpose of this study was to compare pain scores, opioid use, postoperative nausea and vomiting, antiemetic administration and length of hospital stay between postoperative mastectomy patients who received Traditional Recovery After Surgery (TRAS) or the Enhanced Recovery After Surgery (ERAS) protocol.

\section{Methods}

We undertook a retrospective chart review of TRAS and ERAS patients undergoing mastectomy without reconstruction. A statistical power analysis determined a necessary sample size of 102 . Two groups were evaluated: 51 sequential patients having mastectomy without reconstruction before May 2016, prior to ERAS implementation, and 51 sequential patients having mastectomy without reconstruction after implementation of ERAS protocols.

\section{Results}

No significant differences were found between the groups in terms of demographics, pain history, or length of hospital stay.

We found a significant difference between ERAS and TRAS patients:

- Antiemetic use on POD0 ( $20 \%$ vs $41 \% \mathrm{p}=0.018$ )

- Mean Pain Score on PODO

(1.9 vs $3.2 p \leq 0.005)$

- Oral Morphine Equivalent POD0 (26.7 vs $58.3 p \leq 0.005$ )

- Oral Morphine Equivalent POD1 (4.7vs $20.8 p \leq 0.005$ )

\section{Conclusions}

The incorporation of ERAS measures into the surgical care of patients undergoing mastectomy surgery can positively affect postoperative recovery. Our ERAS protocol in patients undergoing mastectomy without reconstruction demonstrated a significant reduction in reported pain, decreased antiemetic use, and significantly reduced the use of opioids in the immediate postoperative period.

\section{Group Characteristics}

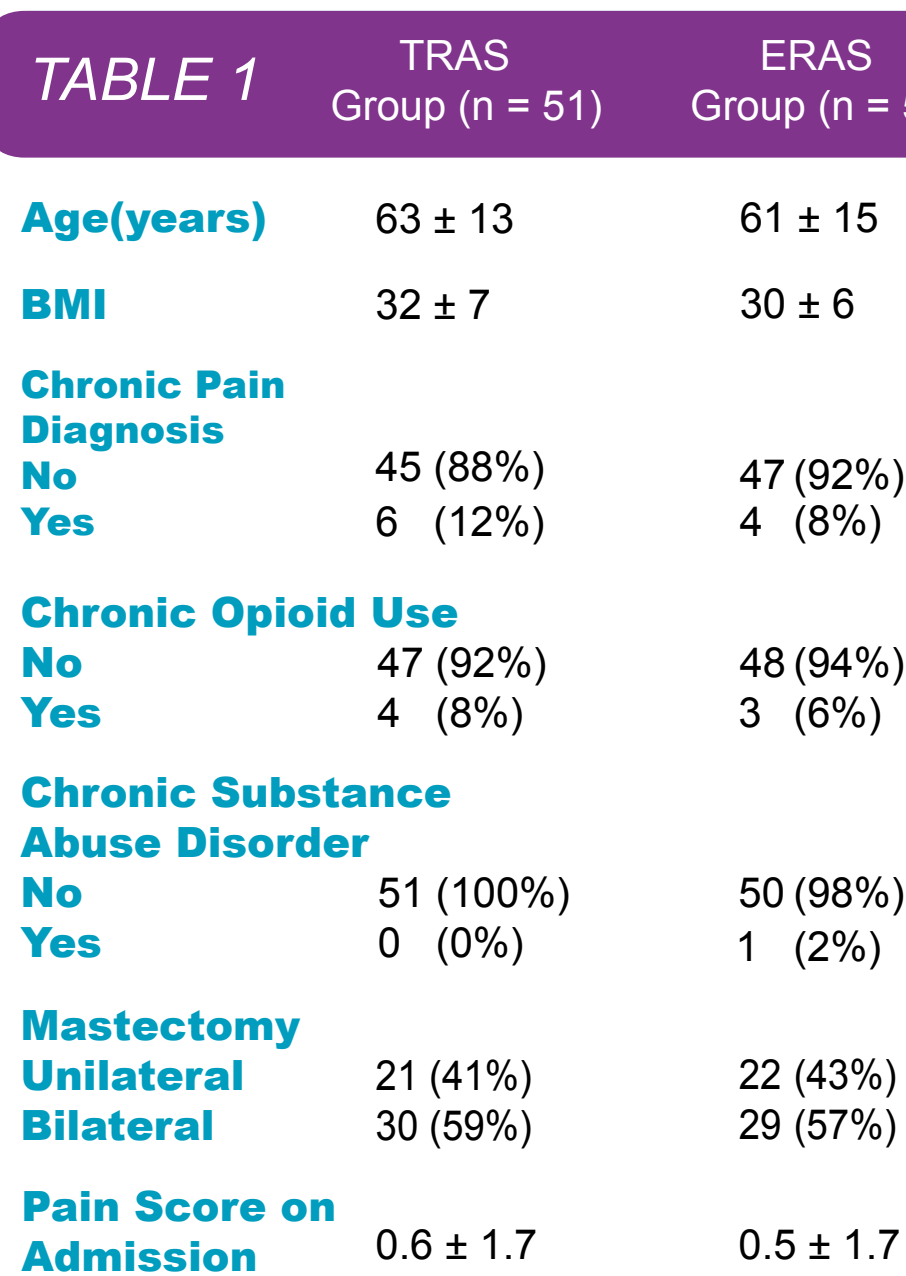

Reported as Mean \pm Standard Deviation or \#(\%)

\section{ERAS Protocol}

Preoperative Protocol

-ERAS education during Pre-Admission

Testing visit

-Acetaminophen 1000mg PO

-Celecoxib 200mg PO

-Pregabalin $75 \mathrm{mg}$ PO

Intraoperative Protocol

-General Anesthesia

- TIVA (Propofol)

-PECS Blocks after induction

-Avoid/Minimize Opioids

-Dexamethasone 4-8mg

- Ondansetron 4mg

-Minimize Excess Fluid Administration

Postoperative Nutrition

-Advance as tolerated the day

of surgery

\section{Analgesia}

-Acetaminophen 1000mg PO Q6hours

- Celecoxib 200mg PO BID

-Carisoprodol or Cyclobenzaprine

PO Q8H PRN muscle spasm

- Tramadol 50mg PO Q6H PRN

VAS 4-6

-Oxycodone 5-10mg PO Q4H PRN

VAS $>7$

-Hydromorphone 0.3-0.5mg IV Q2H

PRN VAS $>7$
Pre \& Post ERAS Mastectomy Group Comparison by Outcome

\begin{tabular}{|c|c|c|c|}
\hline TABLE 2 & $\begin{array}{c}\text { TRAS } \\
\text { Group }(n=51)\end{array}$ & $\begin{array}{c}\text { ERAS } \\
\text { Group }(n=51)\end{array}$ & $\mathrm{p}$ \\
\hline \multicolumn{4}{|c|}{ Length of Operation } \\
\hline $\begin{array}{l}\text { Length of Hospi } \\
\text { (total nights) }\end{array}$ & $\begin{array}{l}\text { Stay } \\
1.1 \pm 0.2\end{array}$ & $1.0 \pm 0$ & .083 \\
\hline $\begin{array}{l}\text { Post-op Antieme } \\
\text { No } \\
\text { Yes }\end{array}$ & $\begin{array}{l}\text { C PODO } \\
30(59 \%) \\
21(41 \%)\end{array}$ & $\begin{array}{l}41(80 \%) \\
10(20 \%)\end{array}$ & $.018^{*}$ \\
\hline $\begin{array}{l}\text { Post-op Antieme } \\
\text { No } \\
\text { Yes }\end{array}$ & $\begin{array}{l}\text { C POD1 } \\
\qquad 4(86 \%) \\
7(14 \%)\end{array}$ & $\begin{array}{l}50(98 \%) \\
1 \quad(2 \%)\end{array}$ & .060 \\
\hline $\begin{array}{l}\text { Post-op Antieme } \\
\text { No } \\
\text { Yes }\end{array}$ & $\begin{array}{l}\text { C POD2 } \\
0 / 3\end{array}$ & $\mathrm{n} / \mathrm{a}$ & \\
\hline $\begin{array}{l}\text { Episodes of Von } \\
\text { (\#) }\end{array}$ & $\begin{array}{l}\text { ing PODO } \\
.02 \pm .14\end{array}$ & $0 \pm 0$ & 1.000 \\
\hline $\begin{array}{l}\text { Episodes of Von } \\
\text { (\#) }\end{array}$ & $\begin{array}{l}\text { ing POD1 } \\
\quad .02 \pm .14\end{array}$ & $0 \pm 0$ & 1.000 \\
\hline $\begin{array}{l}\text { Episodes of Von } \\
\text { (\#) }\end{array}$ & $\begin{array}{l}\text { ting POD2 } \\
0 / 3^{\#}\end{array}$ & $\mathrm{n} / \mathrm{a}$ & \\
\hline Mean Pain Scor & $\begin{array}{l}\text { PODO } \\
\qquad 3.2 \pm 1.3\end{array}$ & $1.9 \pm 1.8$ & $.000^{* *}$ \\
\hline $\begin{array}{l}\text { Mean Pain Scor } \\
\text { Mean Pain Scor }\end{array}$ & $\begin{array}{l}\text { POD1 } \\
2.1 \pm 1.7 \\
\text { PD2 } \\
2.1 \pm 1.1^{\#}\end{array}$ & $\begin{array}{l}1.2 \pm 1.6 \\
\mathrm{n} / \mathrm{a}\end{array}$ & .008 \\
\hline Max Pain Score & $\begin{array}{l}\text { ODO } \\
\quad 7.4 \pm 1.7\end{array}$ & $4.7 \pm 3.2$ & $.000^{* *}$ \\
\hline Max Pain Score & $\begin{array}{l}\text { OD1 } \\
\quad 4 \pm 3\end{array}$ & $2.8 \pm 2.8$ & .029 \\
\hline $\begin{array}{l}\text { Max Pain Score } \\
\text { Oral Morphine E }\end{array}$ & $\begin{array}{l}\text { OD2 } \\
5 \pm 2.6^{\#} \\
\text { uivalent }\end{array}$ & $\mathrm{n} / \mathrm{a}$ & \\
\hline PODO (mg/day) & $58.3 \pm 33.8$ & $26.7 \pm 24.1$ & $.000^{* *}$ \\
\hline $\begin{array}{l}\text { Oral Morphine E } \\
\text { POD1 (mg/day) }\end{array}$ & $\begin{array}{l}\text { uivalent } \\
\qquad 20.8 \pm 19.8\end{array}$ & $4.7 \pm 7.7$ & $.000^{* *}$ \\
\hline $\begin{array}{l}\text { Oral Morphine E } \\
\text { POD2 (mg/day) }\end{array}$ & $\begin{array}{l}\text { uivalent } \\
\qquad 10.8 \pm 11.3^{\#}\end{array}$ & $\mathrm{n} / \mathrm{a}$ & \\
\hline
\end{tabular}

Mobilization

- Out of bed and walking 3 times/day

-Sitting up in chair for all meals

Beyond Discharge Protocol

-Acetaminophen 1000 mg PO Q6

hours $x 48$ hours

- Celecoxib 200 mg PO BID x 48 hours

-Carisoprodol or Cyclobenzaprine

PO Q8H PRN muscle spasm

- Oxycodone 5-10 mg PO Q4H PRN

VAS $>7$ 